\title{
A CLOUD BASED SECURITY FOR ADVANCED MINIMIZING ENERGY BASED DATA SHARING IN MULTI-CLOUDS P.INDRAJA ${ }^{1}$ M.Tech Computer Science, SK.SAIDA ${ }^{2}$ Assoc. Professor Dept. Of CSE SREE VAHINI INSTITUTE OF SCIENCE\&TECHNOLOGY
}

DOI: 10.48047/IJARST/V10/I11/03

\begin{abstract}
:
Protect Cloud is better than protecting your account calculate with internal consumption characteristics assets. In terms of cloud computing, it provides cloud services give an endless storage and file information and creates an abstracts information uploading the data of various clients. It can help customers reduce economic costs of document management by moving with the district administration utility and policies to use on cloud servers. Offer safe statistics The security key can be confiscated Distribute and share information for dynamic groups. Within this task, the existing scheme is able to help Dynamic organizations work effectively when there are new users in case of joining the organization or termination of the user from the organization, the private keys of the opposite party customers do not need to be reimbursed now and do not need to do so date. In addition, the scheme can achieve comfort customer cancellation cannot be a blocked client data files can be retrieved for the first time although they conspired with unbelievers of clouds. To avoid these threats, conspiracies against information are a how to remove a copy of a statistical copy, the cloud has been widely used in garages to reduce storage space, throughput and deployment in cloudy weather information is saved. Recommended compiler The encryption model has been widely followed Convenient anti-conspiracy, correct and reliable control a variety of compiler keys.
\end{abstract}

Keywords: Advanced cloud, S3-Buckets, Access controls, Privacy Preserving Key Distribution and sharing techniques.

\section{Introduction}

The next generation of computing is Cloud computing, in which we provide both centralized computing resources (hardware and software) through a network of centralized resources. Cloud Computing is a huge savings, The processing, applications, operating system, network and various other infrastructures, all the specified functions are centralized in a large server called cloud server. These features come in a variety of forms required by the transmitter and can be accessed through Systems, Mobile, Tabs and other required media. A brief discussion of common cloud applications is a sign of abstraction in complex infrastructure in a central location. Cloud computing provides reliability over long distancesaccess to any media with user data, software, software, security, and computing. Central cloud computing consists of hardware, software, and application resources. Internet and mobile wireless technologies are managed by third-party services. The party and thirdparty users get the opportunity to use the resource in the way they want. These 


\section{International Journal For Advanced Research In Science \& Technology}

A peer reviewed international journal

\section{IJARST}

services often provide access to high-end networks of advanced software applications and server computers. The next generation of computing on the Internet will be cloud computing. With cloud computing, we can reduce infrastructure, maintain huge systems, and do green computing through a single centralized system that provides resource services to a wide range of users. To overcome the disadvantages of investment and maintenance and getting rid of the proposed intruders The architecture is cloud architecture. The following picture shows the structure of cloud computing. Main purpose The purpose of cloud computing is to use traditional methods supercomputing's procedure, supercomputer or In a local area network, we deploy servers and use all servers facilities through a connected network. Local The network server performs highperformance computing In a centralized manner of energy and other basic resources, In general make tens of trillions of calculations inside, user-oriented applications such as finance personal information portfolio, provide a database or large-scale, power supply Computer games. The above technology is the same implemented on cloud computing to expand consumption networks of large servers usually connected Various media internet, Wireless, these servers work at a very low cost to the user's computer or mobile phone specialized connection technology, security and data processing and dissemination of access information. It contains a shared Cloud server and information technology infrastructure connected major systems and resources Collaborate on media sharing.
ISSN: 2457-0362

Virtualization and sharing techniques on the cloud servers use up resources and power cloud computing for a wide range of users or researchers

\section{Literature Survey:}

Cloud computing with internal data exchange and low maintenance allows for better resource utilization. For cloud computing, the cloud service provider offers customers unlimited storage space to store data [1]. It can help customersreduce the financial costs of information management by moving local management systems to cloud servers. However, security is a major concern as we make the information garage more sensitive to cloud vendors.

To keep statistics private, it is not uncommon for clients to encrypt data documents before uploading them to the cloud. Unfortunately, for cloud dynamic companies, for example, it is quite difficult to design a convenient and efficient data sharing scheme. Kallahalla et al [3] initially provided a cryptographic storage system that allowed reliable statistics to be shared on trusted servers based on a file sharing strategy.Encrypt each filegroups and file_group with a document lock key. However, the device has overstated the distribution of keys because it wants the registry keys to be updated and the user wants to pay for the cancellation. Other record sharing schemes

reliable servers are recommended in [4] and [5]. However, the complexity of user participation and cancellation in these schemes increases linearly as the number of data owners and blocked or deletedusers increases. Yu et al [6] uses key policy features such as full policy-based encryption [7], proxy re-encryption, and 


\title{
International Journal For Advanced Research In Science \& Technology
}

\author{
A peer reviewed international journal
}

\section{IJARST}

lazy re-encryption, and integrated strategies are controlled without disclosing the content of the document. However, the single owner method may interfere with the implementation of any program. A member within an organization can use a cloud provider to store and share information documents with others. Liu et al [10] have created a multi-owner, easyto-share information system called Mona. It was stated that the scheme would be available to users who had changed or revoked it, and that they would not be able to access the statistics to be shared again as soon as it was blocked. However, the scheme will suffer from a conspiracy attack through the blocked user and the cloud [13]. The blocked user can use itits private key can be retrieved after decrypting the encrypted data record and deleting the game statistics in a cloud conspiracy. During the document access phase, the blocked user sends the request to the cloud and then to the cloudexamines and responds to the person who returned the relevant encrypted information document and revocation list.

The invalid user can then calculate the encryption key using a set of rules. Eventually, this attack could lead to the sharing of blocked client statistics and the disclosure of various secrets of valid members. Zhou et al [14] allows the use of encrypted information in a cloud garage using a function using a comfy-based encryption method. He claimed that the scheme could invalidate the green people. Integrate function-based functions to manage a convenient encryption policy to store huge amounts of information in the cloud. Unfortunately, the scheme is not vulnerable without inter-enterprise
ISSN: 2457-0362

validation and conspiracy attack. Ultimately, this attack could lead to the disclosure of sensitive statistics. Files Zou et al. [15] proposed a practice and a key vertebral control mechanism based on joint computation. It is intended to exercise its right to govern internationally Get green to run a dynamic business. Unfortunately, the convenient way to share personal perpetual portable secrets is not always supported, and it is possible for an attacker not to divulge a secret as soon as he or she receives it. Nabel et al. [16] suggested that a public cloud-based content sharing scheme be kept private. However, this scheme is not reassuring because it promises securityissuance of ID tokens and implemented advanced sharing policies for next level of security to the cloud data.

\section{Our Proposed System:}

We describe the main objectives of the proposed scheme design in terms of key distribution, information security, access control, and efficiency. Key Distribution: Key distribution requirementsUsers can obtain private management keys from the group manager without any certification. In other existing schemes, the communication channel achieves this goal if it is considered reliable, but according to our scheme, we can achieve it without this strong imagination. Data confidentiality: The confidentiality of information for dynamic groups remainsimportant and difficult issue. In particular, blocked users will not be able to decode the information stored after the cancellation. Benefits: A member of a group can save and share data files with other members of the group. User cancellation can be done without any other intervention, which means that the 


\section{International Journal For Advanced Research In Science \& Technology}

A peer reviewed international journal

\section{IJARST}

remaining users do not need itprivate key update Network-based architecture provides different policies between the data owner, user and cloudadministrator. Cloud admin provides different policies regarding application sharing, uploading, downloading, and storing data based on policy key holders.

\section{Key Exchange mechanism:}

The Group Key integration crypto system includes the Group Key AggregateCryptosystem algorithms [17]. The data owner installs most people using Setup, generates a public / non-public key, and combines the use of KeyGen. Confidential documents are encrypted using the algorithm. The statistician will generate a combination using the master secretkey to decrypt a group of documents.

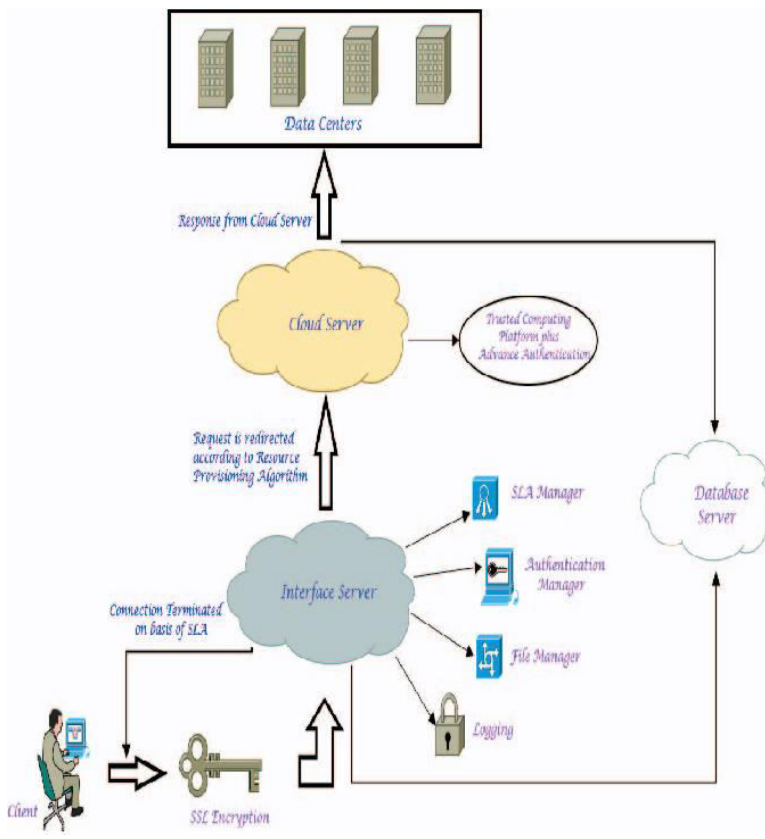

Fig.1 :Cloud Based Key Exchange Architecture

The generated keys can be safely handed over to the delegates (via a calm email or convenient tools). Finally, any user with a mixed key can decode the statistical report
ISSN: 2457-0362

and load it down. Figure 1 shows the structure of the gadget. The state of the users in this structurefor example, 1 person wants to upload a document to the cloud, while user 2 wants to download information from the cloud. When User-1 imports data and files, the information is first encrypted using the DES rule set [14], and after the record is receivedclouds. Separately generated private key. Sharing keys across multiple parties of cloud. Only authorized user can access the key for one time download.

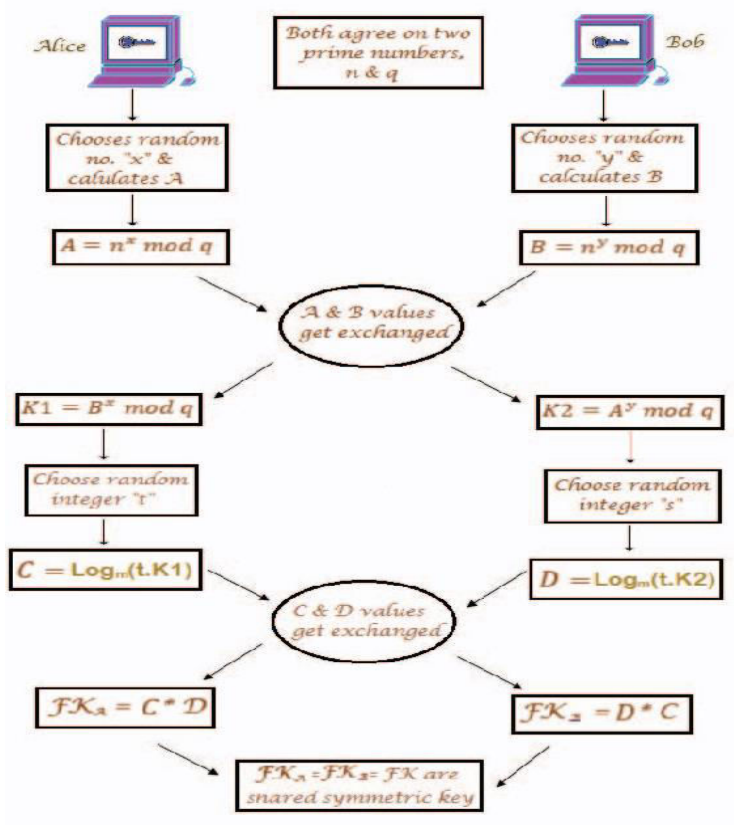

Fig.2 Algorithm Internal Process Flow Diagram

The green building key aggregation method is used to combine non-public keys and create a fixed size key called a mixed key. For the boot module, the receiver can load the recording using the mixing button sent by mail.

with the help of the sender. If the mixed key is legitimate, you will be allowed to download the document. When the recipient downloads the file, the mixed key 


\section{International Journal For Advanced Research In Science \& Technology}

\section{IJARST}

is approved or the extraction is done with non-public keys.

\section{IMPLEMENTATION:}

In this program, the installation is performed by placing and extracting rar documents on a cloud server. The data owner performs the configuration of the account on the server. The most effective configuration package requires covert protectionoption. This segment is made by the registry owner to create a public or master key Pair. This segment is performed by someone who wants to send encrypted information. Encryption and encryption algorithms come in the form of public parameters such as pk and messagesm, and I express the magnificence of the encrypted text. The set of rules encrypts the message and retrieves the encrypted text $\mathrm{C}$ so that the message can be used or used by the authorized user in the most efficient way. This step is completed by the data owner in order to increase the encryption power of the encrypted text in a particular packageThis is done with the help of a candidate authorized to decrypt. KAC is simple and easy to delegate.

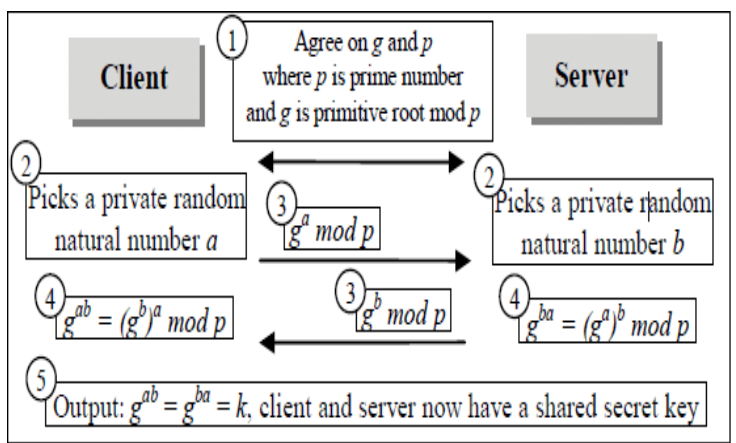

Client and Server Exchanges key based on policies associated in cloud

Algorithm for Encryption

Now here comes an addition in key based agreegation using advanced cloud. In
ISSN: 2457-0362

keyagreegate, check that $\mathrm{Xa}$ is not equal to the identity element $\mathrm{O}$ and its coordinates are otherwise valid ,cipher text enc=A_HASH $(\mathrm{m})$, where hash is the same function used in the signature. Inorder to encrypt a message M1 having i1 ascipher text through key agreegate Algorithm to generate based Aggregate Key tells $\mathrm{S}$ is the set of cipher text indices of those files whose aggregate-key is to be Generated. Following is the code to generate aggregate-key.

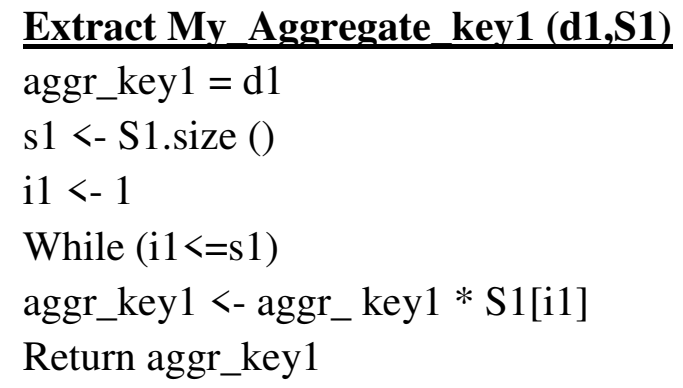

\section{Algorithm for decryption}

Here comes another modification and addition in algorithm.In key based agreegate, check that $\mathrm{Xa}$ is not equal to theidentity element $\mathrm{O}$ and its coordinates are otherwisevalid ,cipher text enc=A_HASH $(\mathrm{m})$, where hash is the samefunction used in the signature. To encrypt a message M1having i1 as cipher text through key agreegateIn ourapproach, to decrypt a set of files whose cipher textindices are kept in set S1, following is the pseudo code of our approach.

\section{Extract_My_Decryption(C1,aggr1} key1,S1)

S1 <- S1.size()

i1 $<-1$

while(S1!=empty)

temp1 $=$ temp $1 * \mathrm{~S} 1[\mathrm{i} 1]$

dec_data_1 = aggr_key1/temp1 i<- 1

while $\mathrm{S} 1$ !=empty 1

derypt1(key1,document1) 


\section{International Journal For Advanced Research In Science \& Technology}

A peer reviewed international journal

\section{IJARST}

We compared the performance modeling with Mona using NS2 [10] and showed the first dynamic radiation encryption (ODBE) scheme [12]. Without losing the commonality, the data representation size is 16 bits, which gives the group capacity of the data files. Similarly, user and group recognition is set to 16 bits. The process for both team members and group managers takes place on Core 2 T5800 2.0 $\mathrm{GHz}$, DDR2 800 2G, and Ubuntu 12.04 $\mathrm{X} 86$ laptops. The cloud process is performed on a Core i7-3630 laptop

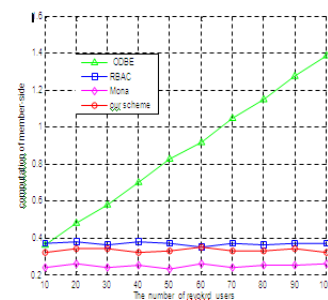

(a) enerating a $10 \mathrm{MB}$ file

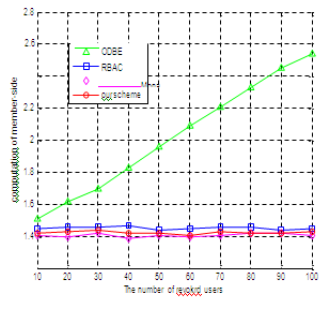

(b) Generating a $100 \mathrm{MB}$ file
Study of Various Algorithms by uploading $10 \mathrm{MB}$ to $100 \mathrm{MB}$ of files

$2.4 \mathrm{GHz}$, DDR3 1600 8G, Ubuntu 12.04 X64. We choose an elliptic curve to order a group of 160 bits. Computational Costs of Members Figure 2 Comparing Different Algorithms in File Uploading As shown in Figure 2, we listed a comparative cost of members for file uploads between ODBE, RBAC, Mona, and our schema. This, of course and observed that the estimated cost of members in our scheme does not depend on the number of invalid users. The reason for this is that in our scheme, the user authentication process is delegated to the group manager, so that legitimate clients encrypt the data file without the involvement of other clients, including both legitimate and non-legitimate ones. Blocked and deleted or blocked customers. Conversely, the computational cost increases with the number of users who are
ISSN: 2457-0362

disabled in ODBE. This is because the client must perform a number of operations, including point multiplication and exponential, to calculate the parameters.

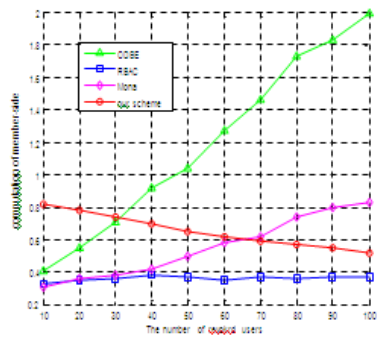

(a) Downloading a $10 \mathrm{MB}$ file

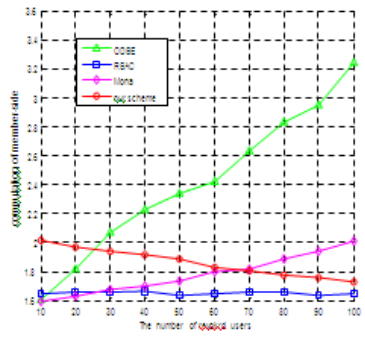

(b) Downloading a $100 \mathrm{MB}$ file

\section{Cost or Computation \\ Conclusion:}

In this document, we have developed anticonspiracy documents The sharing scheme of dynamic companies in the cloud. Inside Under our scheme, customers can securely obtain their clients' non-public keys from the group manager's certification officecommunication channels. And our scheme able to effectively assist dynamic organizations when and where The new user joins the organization or becomes a user blocked, other person's private keys users do not want to recalculate or update.In addition, our scheme allows you to find a comfortable person If blocked or deleted users will not be able to get it as soon as the initial data documents appearalthough they conspired with the cloud of unbelief

References;

[1] М. Армбруст, А. Фокс, Р. Гриффит, A. D. Joseph, R. Katz, A. Конвински, Г. Lee, D. Patterson, A. Rabkin, I. There were Stoika and M. Zakharia. "Cloud Computing Perspectives"Com. ACM, bot. Fifty-three, no. 4, pages 50-58,

April 2010 


\section{International Journal For Advanced Research In Science \& Technology}

ISSN: 2457-0362

[2] S. Kamara and K. Lauter, "Cryptographic Cloud Preservation, "Proc. Fire Conf. Financial cryptography and Information Security (FC), January 2010, No. 136-149.

[3] M. Kallahalla, E. Riedel, R. Svaminathan, Q. Van,K. Fu, "Pluto: a reliable file sharing mode that can be expanded "Unreliable Storage," Proc. USENIX Conf. And FIG Storage technology, pages 29-42, 2003.

[4] E. Goh, H. Shacham, N. Modadugu, and D. Boneh, "Sirius: Maintaining Remote Uncertainty" Proc.Network and Distribution System Security Symp.(NDSS), page 131-one hundred and forty-five, 2003.

[5] G. Ateniese, K. Fu, M. Green, and S. Hohenberger,"Improved Proxy encryption SchemesCommonly used data storage applications, "Proc.Network and Distribution System Security Symp.(NDSS), 2005, 29 forty-three pages. [6] Shuchen Yu, Kong Wang, Kui Ren, and WeijingLu said, "Achieving safe, scalable and sophisticated processingInformation Access Management in Cloud Computing ", Proc. ACMSymbol. Information, Computer, Com. Security, page.282-292, 2010.

[7] V. Goyal, O. Pandey, A. Sakhai, B. Waters,"Attribute-based encryption with sophisticated processing-Track encrypted information, "Proc. ACM settings.Computer and Com. Security (CCS), 2006 89-98

[8] R. Lu, X. Lin, X. Liang, and Shen "SafeProvenence: The basis of bread and butter-Cloud Computational Studies, "Proc. ACM symptoms.Information, Computer, Com. Security, page 282January 292, 2010
[9] B. Waters, "Encrypted, based on policy attributes Encryption: Transparent, efficient and affordableSafeimplementation "Proc. Fire Conf. And FIG 23 theories of public key cryptography. PublicGoal cryptography, http://eprint.Iacr.Org/2008/290.Pdf,2008 [10] Xuefeng Liu, Yuiking Zhang, Boyan Wang, andJingbo Yang, "Mona: It's safe to share information with multiple ownersFor Dynamic Groups in the Cloud. "IEEE transactionsVolume on Parallel and Distribution Systems. 24, no. 6, page.1182-1191, June 2013.

[11] D. Boneh, X. Boyen, and E. Goh. "Fixed character encryptionSchifrext, "Proc. Ann. Fire Conf. And FIGUse of cryptographic methods(EUROCRYPT), pp. 440-456, 2005.

[12] C. Delerablee, P. Paillier, and D. Pointcheval, et al"Complete integration and reliable dynamic radiation encryptionFixed Size Ci-perttexts or DecriptionKey "Proc. First Int Conf. Combination-based cryptography, p.39-59 of 2007. 